\title{
ON A SUBCLASS OF BAZILEVIČ FUNCTIONS
}

\author{
P. J. EENIGENBURG, S. S. MILLER, \\ P. T. MOCANU AND M. O. READE ${ }^{1}$
}

ABSTRACT. The authors show that certain Bazilevič functions are spiral-like. Then the authors study the growth and Hardy classes of those special functions.

Introduction. I. E. Bazilevič [2] gave an explicit construction for a class of functions analytic and univalent in the unit disc $D$ (see also [10]). His result was as follows.

Theorem 1. Let $g$ be univalent starlike in $D$ with $g(0)=0$, and let $h$ be analytic and satisfy $\operatorname{Re}\left(e^{i \lambda} h(z)\right)>0$ in $D$ for some real $\lambda$. Then if $\alpha>0$ and $\beta$ is real, the function

$$
f(z)=\left\{\int_{0}^{z} g^{a}(\zeta) h(\zeta) \zeta^{i \beta-1} d \zeta\right\}^{1 /(\alpha+i \beta)}
$$

is analytic and univalent in $D$.

In this paper we consider the functions $f$ that arise from (1) when $h(z) \equiv 1$. Such a function $f$ must satisfy

$$
\operatorname{Re}\left[1+z f^{\prime \prime}(z) / f^{\prime}(z)+(\alpha-1+i \beta) z f^{\prime}(z) / f(z)\right]>0, \quad z \in D .
$$

Conversely, if $f$ is analytic in $D$, with $f(0)=0, f(z) f^{\prime}(z) / z \neq 0(z \in D)$, and if $f$ satisfies (2) for some $\alpha>0, \beta$ real, then $f$ can be written in the form (1), with $h(z) \equiv 1$. Let us denote the class of such functions $f$ by $B(\alpha+i \beta)$. The class obtained when $\beta=0$ has been studied extensively [5], [6], [7], [8], [9]. The class $B(1+i \beta)$ has recently been considered by $\mathrm{H}$. Yoshikawa [12]; he showed that if $f \in B(1+i \beta)$ then $f$ is $\gamma$-spiral-like, where $\gamma=$ $\operatorname{arc} \tan \beta$. We generalize this to

Received by the editors December 5, 1972.

AMS (MOS) subject classifications (1970). Primary 30A32; Secondary 30A86, $46 \mathrm{~F} 15$.

Key words and phrases. Bazilevič function, univalent, Hardy class, spiral-like, growth.

${ }^{1}$ This author acknowledges support received from the National Science Foundation via NSF Grant GP 28115. 
Theorem 2. If $f \in B(\alpha+i \beta)$ then $f$ is $\gamma$-spiral-like, where $\gamma$ satisfies $\alpha+i \beta=\left(\alpha^{2}+\beta^{2}\right)^{1 / 2} e^{i \gamma},-\pi / 2<\gamma<\pi / 2$.

Proof. Define a function $w$ analytic in $D$ by

$$
e^{i \gamma} \frac{z f^{\prime}(z)}{f(z)}=\cos \gamma\left(\frac{1+w(z)}{1-w(z)}\right)+i \sin \gamma, \quad z \in D \text {. }
$$

One easily checks that $w(0)=0, w(z) \neq \pm 1(z \in D)$. It suffices to show $|w(z)|<1$ for $z \in D$. Let $w(z)=R(z) e^{i \phi(z)}$ for $z=r e^{i \theta}$ and suppose that $z_{0}=r_{0} e^{i \theta_{0}}$ is a point of $D$ such that

$$
\max _{|z| \leq r_{0}}|w(z)|=\left|w\left(z_{0}\right)\right|=1
$$

Then $\left.(\partial R / \partial \theta)\right|_{z=z_{0}}=0$, and since

$$
\frac{z w^{\prime}(z)}{w(z)}=\frac{\partial \phi}{\partial \theta}-i \frac{1}{R} \frac{\partial R}{\partial \theta}
$$

we have at the point $z_{0}$,

$$
z_{0} w^{\prime}\left(z_{0}\right) / w\left(z_{0}\right)=\left.(\partial \phi / \partial \theta)\right|_{z=z_{0}} .
$$

We shall now show that

$$
\operatorname{Re}\left[1+z f^{\prime \prime}(z) / f^{\prime}(z)+(\alpha-1+i \beta) z f^{\prime}(z) / f(z)\right]_{z=z_{0}}=0
$$

thus contradicting the assertion $f \in B(\alpha+i \beta)$. By (3), (4) can be written as

$$
\operatorname{Re}\left[\frac{z P^{\prime}(z)}{P(z)+i \tan \gamma}+\left(\alpha^{2}+\beta^{2}\right)^{1 / 2}(\cos \gamma P(z)+i \sin \gamma)\right]_{z=z_{0}}=0
$$

where $P(z)=(1+w(z)) /(1-w(z))$. Since $\left|w\left(z_{0}\right)\right|=1$ and since $\left[z_{0} w^{\prime}\left(z_{0}\right) / w\left(z_{0}\right)\right]$ is real, it follows that $P\left(z_{0}\right)$ is imaginary and $z_{0} P^{\prime}\left(z_{0}\right)$ is real. Hence (5) holds at $z_{0}$. This completes the proof.

Theorem 3. If $\alpha^{\prime}+i \beta^{\prime}=t(\alpha+i \beta), t \geq 1$, then $B(\alpha+i \beta) \subset B\left(\alpha^{\prime}+i \beta^{\prime}\right)$.

Proof. Since $f$ is $\gamma$-spiral-like $\left(\alpha+i \beta=\left(\alpha^{2}+\beta^{2}\right)^{1 / 2} e^{i \gamma}\right)$,

$$
\operatorname{Re}\left[(t-1)\left(\alpha^{2}+\beta^{2}\right)^{1 / 2} e^{i \gamma} z f^{\prime}(z) / f(z)\right] \geq 0, \quad z \in D .
$$

Then

$$
\begin{aligned}
& \operatorname{Re}\left[1+z f^{\prime \prime}(z) / f^{\prime}(z)+\left(\alpha^{\prime}-1+i \beta^{\prime}\right) z f^{\prime}(z) / f(z)\right] \\
&= \operatorname{Re}\left[1+z f^{\prime \prime}(z) / f^{\prime}(z)+(\alpha-1+i \beta) z f^{\prime}(z) / f(z)\right] \\
& \quad+\operatorname{Re}\left[(t-1)\left(\alpha^{2}+\beta^{2}\right)^{1 / 2} e^{i \gamma} z f^{\prime}(z) / f(z)\right] \geq 0, \quad z \in D .
\end{aligned}
$$


In the integral representation for functions in $B(\alpha+i \beta)$, namely,

$$
f(z)=\left\{\int_{0}^{z} g^{a}(\zeta) \zeta^{i \beta-1} d \zeta\right\}^{1 /(\alpha+i \beta)},
$$

let us denote by $f_{a+i \beta}$ the function obtained by letting $g$ be the Koebe function $z /(1-z)^{2}$. The following theorem illustrates the dependence of the growth of $f$ on the parameters $\alpha$ and $\beta$.

Theorem 4. Suppose $f \in B(\alpha+i \beta)$.

(A) If $0<\alpha \leq 1 / 2$, then, unless $f$ is a rotation or magnification of $f_{1 / 2+i \beta}, f$ is bounded.

(B) If $\alpha>1 / 2$ and $f$ is not a rotation or magnification of $f_{\alpha+i \beta}$, then there exists $\epsilon=\epsilon(f)>0$ such that $f \in H^{\lambda+\epsilon}$ and $f^{\prime} \epsilon H^{(\lambda /(1+\lambda))+\epsilon}$, where $\lambda=\left(\alpha^{2}+\beta^{2}\right) / \alpha(2 \alpha-1)$.

(C) For $\alpha>1 / 2$ the function $f_{a+i \beta}$ belongs to $H^{p}, \forall p<\lambda$, but does not belong to $H^{\lambda}$.

Proof. Following Sheil-Small's construction [11] of $f$ in "analytic stages" from the representation (6), we set

$$
F(z)=\left(\frac{g(z)}{z}\right)^{\alpha}=\sum_{n=0}^{\infty} C_{n} z^{n}
$$

for a suitable branch of the nonvanishing function $(g(z) / z)^{\alpha}$. Let

$$
G(z)=\sum_{n=0}^{\infty} \frac{C_{n}}{n+\alpha+i \beta} z^{n},
$$

so that $G$ is analytic in $D$ and satisfies the differential equation

$$
(\alpha+i \beta) G(z)+z G^{\prime}(z)=F(z) \text {. }
$$

Sheil-Small [11] shows that $G(z) \neq 0$ in $D$. We now define $f$ by the formula

$$
f(z)=z[G(z)]^{1 /(\alpha+i \beta)} \text {. }
$$

One can easily verify that apart from a constant factor, this defines an analytic branch of the formula (6). By (9) we may write

$$
G(z)=\left[(f(z) / z)^{1+i \beta / a}\right]^{\alpha}=[s(z) / z]^{a},
$$

where $(10)$ is the defining equation for $s$. Since $f$ is $\gamma$-spiral-like (Theorem 2 ), it follows easily that $s$ is starlike in $D$. From (8) we have

$$
z G^{\prime}(z)=(g(z) / z)^{\alpha}-(\alpha+i \beta)(s(z) / z)^{\alpha} .
$$

If $g$ is not a rotation of the Koebe function, then there exists $\epsilon=\epsilon(g)>0$ such that $g \in H^{1 / 2+\epsilon}$ [4]. Furthermore, it is easy to see from (7) that $s$ cannot be a rotation of the Koebe function. Thus, $G^{\prime} \in H^{1 /(2 a)+\epsilon}, \epsilon$ denoting 
a positive number, not necessarily the same in each instance. Hence, if $0<a \leq 1 / 2, G$ is bounded and so $f$ is bounded, by (9). Whence, (A) is proved. For $a>1 / 2$, a Hardy-Littlewood theorem [3, p. 88] shows that $G \in H^{1 /(2 a-1)+\epsilon}$; hence, from (10), $s \in H^{a /(2 a-1)+\epsilon}$. From the identity

$$
(f(z) / z)^{1+i \beta / a}=s(z) / z,
$$

we have

$$
\left|\frac{f(z)}{z}\right|=\left|\frac{s(z)}{z}\right|^{a^{2}\left(\alpha^{2}+\beta^{2}\right)^{-1}} \cdot \exp \left[\frac{\alpha \beta}{\alpha^{2}+\beta^{2}} \arg \left(\frac{s(z)}{z}\right)\right] .
$$

Since $s \in H^{\alpha /(2 a-1)+\epsilon}$ and the exponential factor is bounded, it follows that

$$
f \in H^{\lambda+\epsilon}, \quad \lambda=\frac{\alpha^{2}+\beta^{2}}{\alpha(2 \alpha-1)} .
$$

To complete the proof of (B), we must show the existence of an $\epsilon>0$ such that $f^{\prime} \in H^{\lambda(1+\lambda)^{-1+\epsilon}}$. By Theorem 2, there exists $h, \operatorname{Re}(h(z))>0$ in $D$, such that

$$
e^{i \gamma} z f^{\prime}(z)=f(z) h(z) \text {. }
$$

Fix $\epsilon$ in (14), and for small positive $\delta$, let

$$
k=k(\delta)=(\lambda+\epsilon)(1+\lambda+\epsilon+\delta \lambda+\delta \epsilon)^{-1} \text {. }
$$

Choosing $p=(\lambda+\epsilon) k^{-1}, q=(1+\delta)^{-1} k^{-1}$, and applying Hölder's inequality to (15) with conjugate indices $p$ and $q$, it follows that

$$
\int_{-\pi}^{\pi}\left|f^{\prime}(z)\right|^{k} d \theta \leq\left(\int_{-\pi}^{\pi}\left|\frac{f(z)}{z}\right|^{k p} d \theta\right)^{1 / p}\left(\int_{-\pi}^{\pi}|b(z)|^{k q} d \theta\right)^{1 / q} .
$$

By (14) and the fact that $k q<1$, we have that $\int_{-\pi}^{\pi}\left|f^{\prime}(z)\right|^{k} d \theta$ remains bounded as $r \rightarrow 1$. Hence, the proof of (B) is complete if we show the existence of $\delta>0$ such that $k=k(\delta)>\lambda(1+\lambda)^{-1}$. But this is easily checked by consideration of (16).

Finally, we leave the verification of $(C)$ to the reader.

Remark 1. If we take $\beta=0$ in Theorem 4 , the result is the same as that obtained in [5].

Remark 2. Note that if we divide (2) by $\left(\alpha^{2}+\beta^{2}\right)^{1 / 2}$ and let $\alpha+i \beta \rightarrow \infty$ along the ray $t e^{i \gamma}$ the classes $B(\alpha+i \beta)$ "tend" to the full class of $\gamma$-spiral-like functions. If we also let $\alpha+i \beta \rightarrow \infty$ in Theorem 4 , we find that $\lambda=\lambda(\alpha, \beta) \rightarrow\left(2 \cos ^{2} \gamma\right)^{-1}$; and this is precisely the Hardy class result 
previously known for $\gamma$-spiral-like functions [1].

Remark 3. It is interesting to observe that the level curves of $\lambda=$ $\left(\alpha^{2}+\beta^{2}\right)[\alpha(2 \alpha-1)]^{-1}$ are the right branches of certain hyperbolas that are symmetric with respect to the real line; and they converge on the vertical line $a=1 / 2($ as $\lambda \rightarrow \infty)$. For instance, if $\lambda=1$ we obtain the right branch of the hyperbola $4(\alpha-1 / 2)^{2}-4 \beta^{2}=1$.

The authors wish to thank John Harrington for pointing out an error in the proof of Theorem 2.

\section{REFERENCES}

1. T. Basgöze and F. R. Keogh, The Hardy class of a spiral-like function and its derivative, Proc. Amer. Math. Soc. 26(1970), 266-269. MR 41 \#680.

2. I. E. Bazilevič, On a case of integrability in quadratures of the LoewnerKufarev equation, Mat. Sb. 37(79) (1955), 471-476. (Russian) MR 17, 356.

3. P. L. Duren, Theory of $H^{p}$ spaces, Pure and Appl. Math., vol. 38, Academic Press, New York, 1970. MR 42 \#3552.

4. P. J. Eenigenburg and F. R. Keogh, The Hardy class of some univalent functions and their derivatives, Michigan Math. J. 17(1970), 335-346.

5. P. J. Eenigenburg and S. S. Miller, The $H^{p}$ classes for arconvex functions, Proc. Amer. Math. Soc. 38(1973), 558-562.

6. S. S. Miller, Distortion properties of alpha-starlike functions, Proc. Amer. Math. Soc. 38(1973), 311-318.

7. S. S. Miller, P. Mocanu and M. O.Reade, All a-convex functions are univalent and starlike, Proc. Amer. Math. Soc. 37(1973), 553-554.

8. P. Mocanu, Une propriété de convexité généralisée dans la théorie de la repprésentation conforme, Mathematica (Cluj), 11(34)(1969), 127-133. MR 42 \#7881.

9. P. Mocanu and M. O. Reade, On generalized convexity in conformal mappings, Rev. Roumaine Math. Pures Appl. 16(1971), 1541-1544.

10. C. Pommerenke, Über die Subordination analytischer Funktionen, J. Reine Angew. Math. 218(1965), 159-173. MR 31 \#4900.

11. T. Sheil-Small, On Bazilevič functions, Quart. J. Math. Oxford Ser. (2) 23(1972), 135-142. MR 45 \#8847.

12. H. Yoshikawa, On a subclass of spiral-like functions, Mem. Fac. Sci. Kyu shu Univ. Ser. A 25(1971), 271-279.

DEPARTMENT OF MATHEMATICS, WESTERN MICHIGAN UNIVERSITY, KALAMAZOO, MICHIGAN 49001

DEPARTMENT OF MATHEMATICS, ST ATE UNIVERSITY OF NEW YORK, BROCKPORT, NEW YORK 14420

DEPARTMENT OF MATHEMATICS, THE BABES BOLYAI UNIVERSITY, CLUJ, ROMANIA 48104

DEPARTMENT OF MATHEMATICS, UNIVERSITY OF MICHIGAN, ANN ARBOR, MICHIGAN 